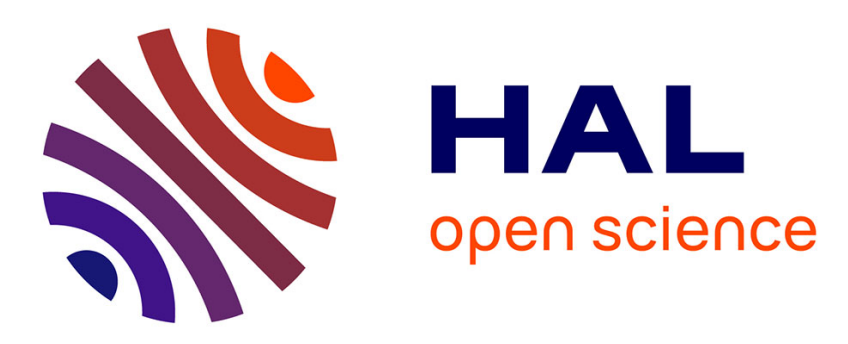

\title{
Gaseous and solid emboli differentiation using radiation force
}

Grégory Souchon, Jean-Marc Girault, Mathieu Biard, Denis Kouamé, François

Tranquart

\section{- To cite this version:}

Grégory Souchon, Jean-Marc Girault, Mathieu Biard, Denis Kouamé, François Tranquart. Gaseous and solid emboli differentiation using radiation force. IEEE International Ultrasonics Symposium (IUS 2005), IEEE- UFFC Society, Sep 2005, Rotterdam, Netherlands. pp.2070-2073, 10.1109/ULTSYM.2005.1603287 . hal-03149788

\author{
HAL Id: hal-03149788 \\ https://hal.science/hal-03149788
}

Submitted on 24 Feb 2021

HAL is a multi-disciplinary open access archive for the deposit and dissemination of scientific research documents, whether they are published or not. The documents may come from teaching and research institutions in France or abroad, or from public or private research centers.
L'archive ouverte pluridisciplinaire HAL, est destinée au dépôt et à la diffusion de documents scientifiques de niveau recherche, publiés ou non, émanant des établissements d'enseignement et de recherche français ou étrangers, des laboratoires publics ou privés. 


\title{
Gaseous and solid emboli differentiation using radiation force
}

\author{
Gregory Souchon, Jean-Marc Girault, Member, Mathieu Biard, IEEE, Denis Kouamé, Member, IEEE \\ and François Tranquart*
}

\begin{abstract}
Cerebral embolisms represent a major part of all ischemic strokes in western countries. It has been found that a good knowledge of emboli (detection, differentiation and sizing), which are foreign particles to blood normal cerebral circulation, remains a promising challenge. Their characterization can be used, in fact, as a diagnostic or therapeutic tool. The most commonly used tool to detect emboli is a transcranial Doppler system. Clinical studies have shown that in the embolic Doppler signature of patient having a prosthetic heart valve present a high frequency modulation index while for patient having atherosclerotic plaque the frequency modulation index is low. We hypothesis that this high level modulation index is probably induced by the radiation force effect (trajectory modification). We will show in this study that it is possible, by using the radiation force, to discriminate gaseous to particulate emboli.
\end{abstract}

Keywords - Transcranial Doppler, emboli discrimination, radiation force, frequency modulation index.

\section{INTRODUCTION}

More $\mathrm{T}$ han two-thirds of all ischemic strokes are reign particles of different nature : blood clot, fat particles or gas bubbles (see the illustration in figure (1)). With a view to produce an accurate diagnostic and a suitable therapy, it is essential to quickly establish the nature of detected emboli. Size of the emboli is equally an important characteristic. Usually, transcranial Doppler systems are used for this purpose. In well-controlled in vitro experiments, the analysis of the Doppler signal can provide some interesting informations concerning the characteristics and the size of emboli. However an accurate characterization in clinical practice is not yet possible by using current technologies.

Many studies, dedicated to differentiate solid to gaseous emboli, have been published in scientific and clinical journals. Most of parameters which are implemented in "onor off-line" systems are based on the linearity of the backscattered Doppler signal. The most popular are the embolic duration (ED), the embolic velocity (EV), the embolus to blood ratio EBR at the emitting frequency, the frequency modulation index [1], the sample volume length (SVL) [2]. Another parameters such as the signal to error ratio (SER) [3], the frequency band and the symmetry of the HITS signature can be used [4].

Clinical studies have shown that in the embolic Doppler signal of patient having a prosthetic heart valve present a high frequency modulation index whereas for patient ha-

The authors work at François Rabelais University, LUSSI FRE CNRS, Tours, France. * INSERM U619, Bretonneau Hospital, Tours, France. E-mail : gregory.souchon@med.univ-tours.fr.

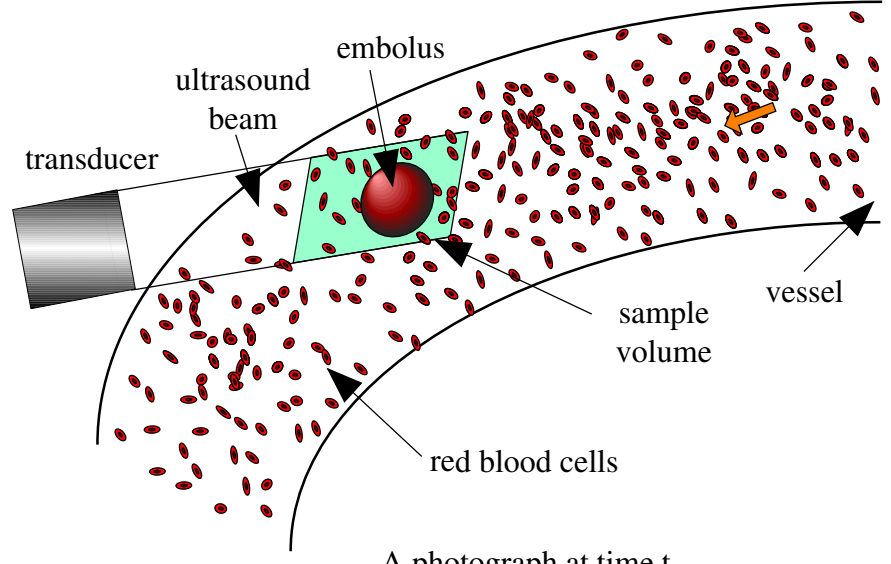

A photograph at time $\mathrm{t}$

Fig. 1.

Sketch of a solid embolus in the sample volume.

ving atherosclerotic plaque the frequency modulation index is low. The Leicester group [1] has provided some realistic explanations : artery curvature, helical flow, harmonic generation and phase cancellation.

By providing another explanation of this strange timefrequency signatures, our study complets and extends the Leicester group results. In fact we hypothesis that the frequency modulation index (FMI), i.e. the slope of the timefrequency signature, is probably induced, at the beginning of the process, by the radiation force effect (trajectory modification). By providing a complementary explanation of this strange frequency modulation, we propose, in the same time, another way to discriminate gaseous to solid emboli. The principle of this new method is to induce controlled or coded displacements of emboli in the sample volume. These coded displacements are simply obtained by setting at different levels the amplitude of the pulses at each pulse repetition period.

Here, in this study we only show the feasibility of this method. A complet study will be the subject of another paper.

\section{Radiation Force and Simulation}

In most cases, radiation force is due to the modification of the energy density of the incident acoustic field. This force emerges from the interaction between the ultrasound beam and the insonified medium. Radiation force appears during the propagation process in the surrounding medium but also for a high contrast of the acoustic im- 


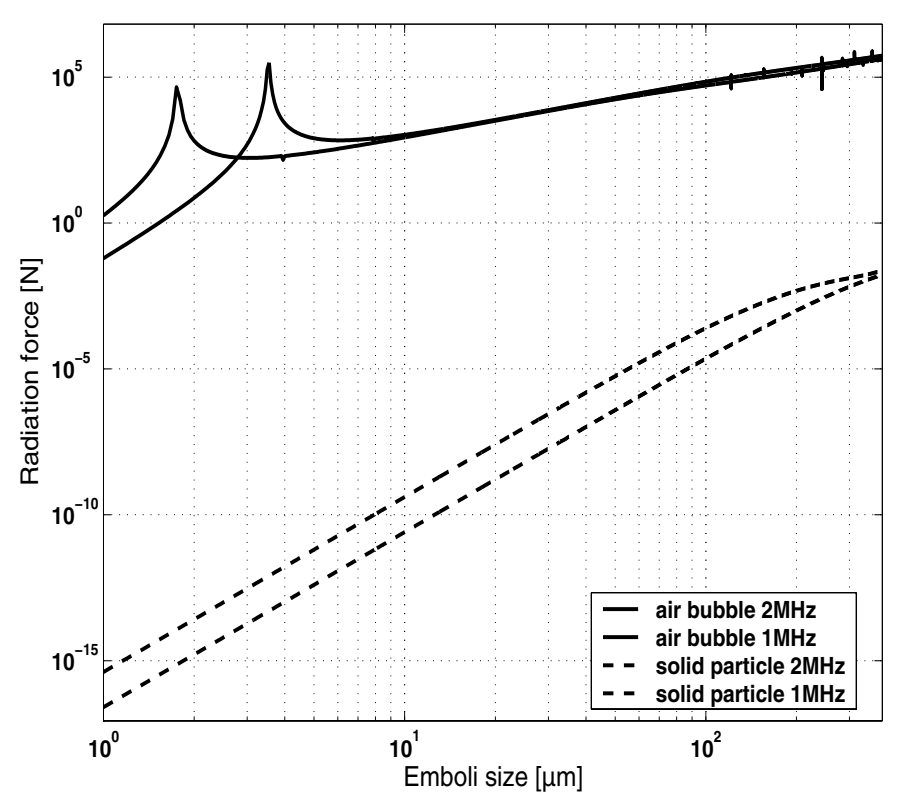

Fig. 2.

Radiation force acting on gaseous and solid spheres with ultrasound frequencies 1 and $2 \mathrm{MHz}$

pedance between the surrounding media and the embolus. By assuming emboli as spheres, the radiation force acting on emboli is given by the Yosioka et al model's [5] and [6]. Theses models do not take into account the propagation phenomenon but only considers the first order scattering. The radiation force is obtained by averaging on time and space the total pressure field. Simulations (see figure (2)) are limited to embolus ranging from $1 \mu \mathrm{m}$ to $400 \mu \mathrm{m}$ (ranging size encountered in clinical investigations). Simulations are made with two ultrasound frequencies : $1 \mathrm{MHz}$ and $2 \mathrm{MHz}$. From figure (2), we can note that the radiation force with respect to the frequency is similar to the backscattered differential cross section of a sphere. From $10 \mu \mathrm{m}$ to $400 \mu \mathrm{m}$, radiation force acting on bubbles is independent of the frequency used (same radiation force level for the two frequency ranges). In figure (2), we can observe that the radiation force acting on a gaseous embolus is always greater than the one acting on a solid particle. We can note the presence of a resonance peak for bubble only in contrast to solid particle. For all the size range and for the two ultrasound frequencies, a threshold set at $0.1 \mathrm{~N}$ allows to discriminate gaseous to solid emboli. In fact, we do not observe directly the radiation force, but rather its effect on the bubble : modification of its trajectory in the artery. The "extra-displacement" or rather the "extra-acceleration" of the embolus due to the radiation force is governed by the Newton's law. Before writing the Newton's law, we precise here that we mean by "extra-acceleration", an additional acceleration of the natural acceleration induced by the spatiotemporal blood flow in which the embolus is embedded. The Newton's law writes :

$$
\sum \vec{F}=\overrightarrow{F_{R}(x, z)}+\overrightarrow{F_{D}(x, z, t)}=m \vec{\gamma},
$$

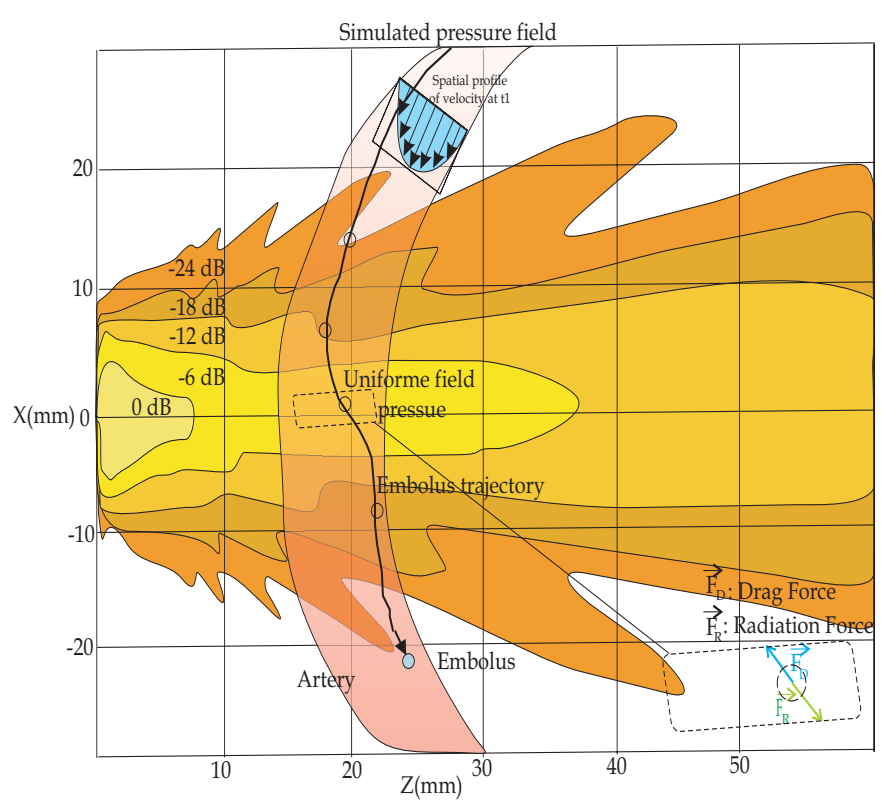

Fig. 3.

Pressure field simulation in a blood vessel.

where $\overrightarrow{F_{R}(x, z)}$ is the radiation force induced by the acoustic contrast between the blood and the embolus and by the propagation up to the embolus. $\overrightarrow{F_{D}(x, z, t)}$ is the resistance force (the well known Drag force). This force is induced whether there is a relative velocity between a particle and the viscous fluid in which the particle is immersed. When an embolus is subject to the radiation force, this one accelerates according to the Newton's law. The acceleration induces a non negligible relative velocity between emboli and fluid, which induces a Drag force. This resistance force depends (in the Newtonian fluid assumption) on the fluid kinematic viscosity, the velocity of the fluid, the velocity of the embolus, the Reynolds number and the Drag coefficient (proportional to the fluid viscosity and the embolus size). $\vec{\gamma}$ and $m$ are the acceleration and the mass of the embolus, respectively. Note that a similar formulation has be done by the Florence Group [7] in the case of contrast agent study. Furthermore, it is important to note that this force is hardly dependent of the spatial velocity distribution (laminar or plug profile) and of the time distribution of the velocity (systole to diastolic phase). In figure (3), we show the pressure map. It is clear that for one embolus travelling at a constant velocity (on a velocity line of the spatial velocity profile) but situated at different positions will be subject to different radiation force due to the non uniformity of the field pressure. For a small area where the field is locally uniform (see figure (3)), the induced "extra-displacement" over the particle will not remain constant because of the velocity lines which are spatially different in the case of a laminar flow. Therefore very strange "extra-acceleration" can be obtained, depending on the equilibrium between the radiation force, the Drag Force, the spatiotemporal blood velocity, the Doppler angle and the spatial pressure level in 


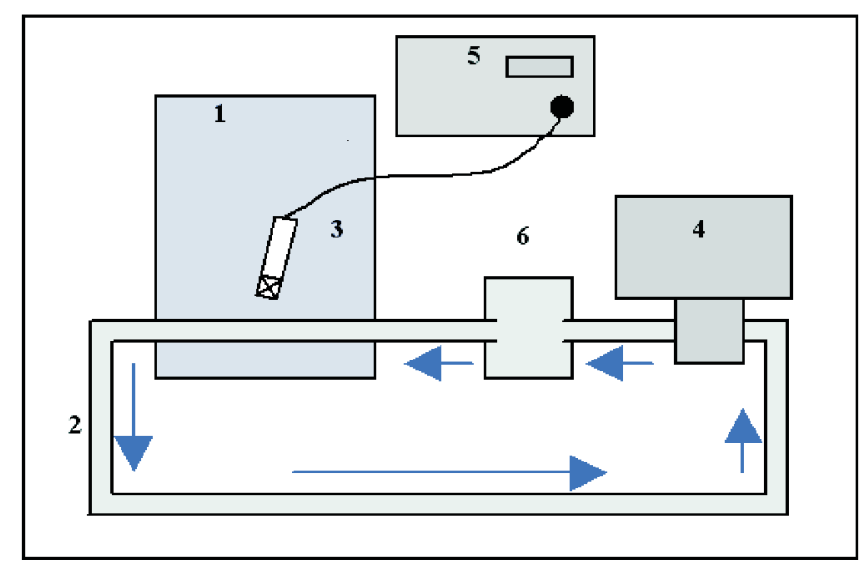

Fig. 4 .

Schematic diagram of the experimental setup.

the sample volume.

Notice that since the mass of a bubble (gaseous emboli) is always less than the mass of a solid particle (of the same size), the "extra-displacement" induced by the radiation force acting on a bubble will be always greater than the one obtained for a solid particle. Consequently, the "extraacceleration" can be identified in the time-velocity/Doppler frequency representation by mean of the instantaneous Doppler frequency slope, i.e. the FMI.

This seems to be a theoretically suitable tool for emboli differentiation and must be evaluated in in vitro experiments. This is presented in the next section.

\section{Material AND Methods}

\section{A. Experimental Acoustical Setup}

A schematic diagram of the experimental set up is presented on the figure (4). A gear pump (4) induces a degassed water circulation in a tygon tube (2). This tube crosses a water tank (1) where a transcranial Doppler transducer (3) is immerged. Circulating emboli are detected by the pulse wave (PW) Doppler system (5). We proposed two types of configurations : without or with skull between the tube and the probe. The transcranial Doppler transducer is an unfocused Vermon probe of central frequency $2 \mathrm{MHz}$. The PW Doppler system, DOP248 Ultrasons Technologies, operates at $2 \mathrm{MHz}$ with pulse of 20 cycles and pulse repetition frequency (PRF) of $8.33 \mathrm{kHz}$. For the highest power delivered by our Doppler system, the pressure level in the tube is $25 \mathrm{kPa}$ without skull and $5 \mathrm{kPa}$ with skull. Gaseous emboli are naturally produced by the gear pump and their size are less than $100 \mu \mathrm{m}$. Solid emboli are peace of pork meal with sizes ranging from $100 \mu \mathrm{m}$ to $300 \mu \mathrm{m}$. The speed of the fluid in the tube is set to a constant value insure us no acceleration. Solid emboli are injected into the secondary water tank (6) and suck in the tube. We used peace of pork meal to simulate solid emboli because we wanted solid emboli close to those observed in clinical studies. Gaseous and solid emboli are detected when they cross the acoustic
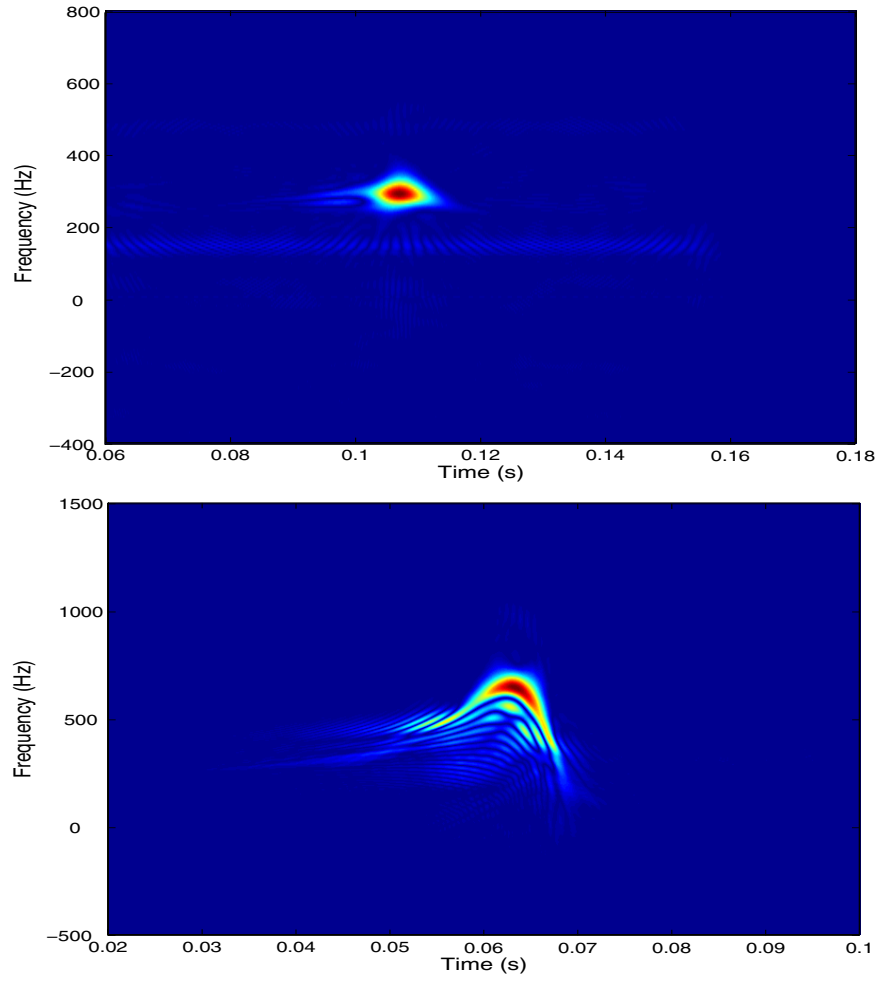

Fig. 5.

Time frequency representation of the Doppler signal in the case of solid emboli (upper) and gaseous emboli (lower), no skull, frequency $2 \mathrm{MHz}$.

beam. Each embolic signal is acquired on a computer. A post processing is applied to these signals to display the frequency modulation index.

\section{B. Signal Post Processing}

As already describe, emboli will be accelerated or decelerated by the radiation force. This change of velocity induces a frequency modulation of the Doppler spectrum. For the two different configurations (with or without skull) and for each emboli we compute the time-frequency representation (Wigner-Ville) of the Doppler signal. The FMI is determined by applying a linaer regression on the instantaneous Doppler frequency.

\section{Results}

\section{A. Solid emboli}

Figure (5a) shows the time-frequency representation of the embolic Doppler signal without skull. The FMI is low but the mean value is around $197 \mathrm{~Hz} / \mathrm{s}$ involving a small "extra-displacement". With skull, no "extra-displacement" is observed.

\section{B. Gaseous emboli}

Figure (5b) show the time-frequency representation of embolic Doppler signal without skull. We can observe a strong frequency modulation of the Doppler spectrum. The 
frequency index modulation is around $20000 \mathrm{~Hz} / \mathrm{s}$. We assume that the gaseous emboli is strongly "extra-displaced" by crossing the ultrasound beam. With skull, the mean value of the frequency modulation index is $772 \mathrm{~Hz} / \mathrm{s}$ involving a weak but detectable "extra-displacement".

The presented results are very interesting in the sense that we can induce a sufficient radiation force to extradisplace an embolus through a skull. We think that the authors in [8], [9], have shown for the first times that it is possible to induce a bubble displacement by means of the radiation force through a human skull.

\section{Discussion}

Frequency modulation index for all gaseous and solid emboli with or without skull are shown on the figure (6). As forecasted, the FMI obtained for gaseous emboli are always greater than for solid emboli. These measures corroborate the feasibility phase previously introduced. The discriminating threshold can be set :

- to $1000 \mathrm{~Hz} / \mathrm{s}$ without skull;

- to $100 \mathrm{~Hz} / \mathrm{s}$ with skull.

By using those thresholds, the false alarm probability is very low. Consequently as reported by [1], the FMI seems to be a suitable tool to discriminate gaseous to solid emboli. The large dispersion of the FMI values is probably due to the dispersion size of emboli and the low number of available data. The emboli "extra-displacement" which is observed through the FMI depends on :

- the nature of the emboli;

- the emboli initial position in sample volume;

- the shape of the artery;

- the spatiotemporal profile of the blood velocity;

- and the non uniformity of the ultrasound beam.

In our in vitro study, the method presented here allows to determine the nature of the emboli. In fact, in vitro studies do not well reproduce all the in vivo encountered cases. For example, if a gaseous embolus crosses a small part of the sample volume, the radiation force effect will involve a low FMI. This can produce a high false alarm probability. By comparing the estimated "extra-displacement" (via the FMI estimation) to the emitting of sequential coded amplitude (different amplitude at each pulse repetition period), it is then possible to reduce the false alarm rate.

As part of collaboration with the Department of Electronic and Telecommunication of Florence (P. Tortoli), we have performed the same experimentation with the MCMG system (Multi Channel and Multi Gate Doppler system). This promising system allowing a depth-time representation permits to measure directly the displacement of a scatter at different depth. The data processing is in progress.

\section{Conclusion}

The used of the FMI seems to be a interesting tool to differentiate gaseous to solid emboli. This feasibility study has to be confirmed by the in vitro study performed with the MCMG system. To reduce the false alarm rate, an automatic amplitude and frequency coding will be developed.

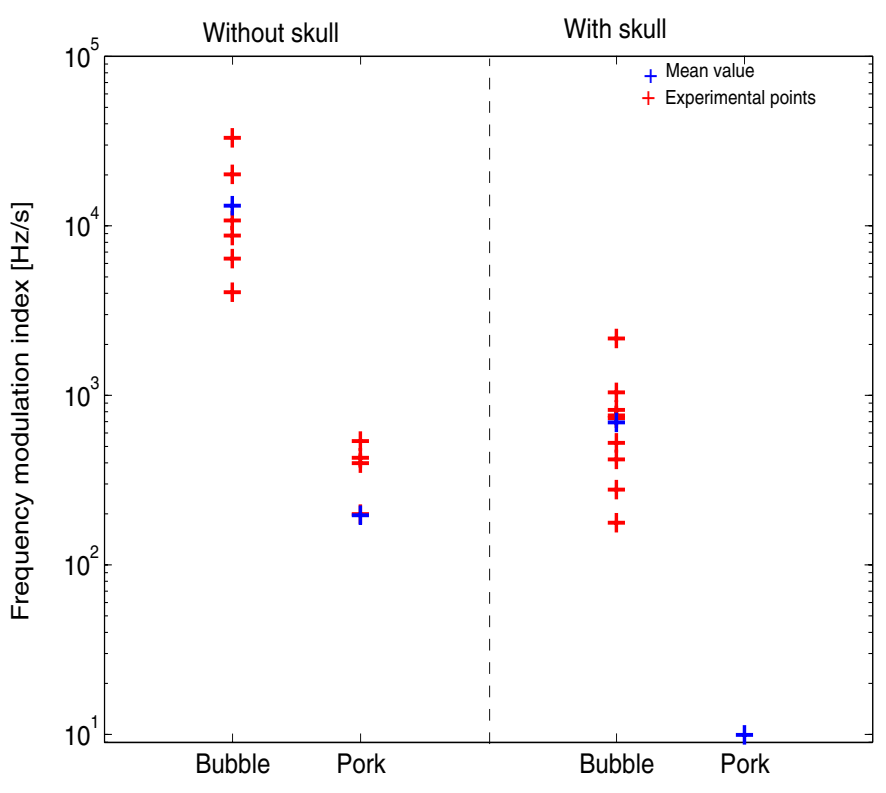

Fig. 6 .

Frequency modulation index for gaseous and solid particle with or without skull.

\section{Acknowlegdement}

This study has been supported by the European community (UMEDS Project) and by the French government (CASC Project).

\section{REFERENCES}

[1] J. Smith, D. Evans, and R. Naylor, "Analysis of the frequency modulation present in doppler ultrasound signal may allow differentiation between partiulate and gaseous cerebral emboli," $\mathrm{Ul}$ trasound in medecine and biology, vol. 23, pp. 727-734, 1997.

[2] J. Smith, D. Evans, P. Bell, and R. Naylor, "A comparison of four methods for distinguishing doppler signals from gaseous and particulate emboli," Stroke, vol. 29, pp. 1133-1138, 1998.

[3] G. Devuyst, J-M. Vesin, P-A. Despland, and J. Bogousslavsky, "The matching pusruit method : a new method of characterizing micro-emboli signals?," Ultrasound in medecine and biology, vol. 26, pp. 1051-1056, 2000

[4] G. Devuyst, G.A Darbellay, J-M. Vesin, V. Kemeny, M. Ritter D.W. Droste, C. Moline, J. Serena, R. Sztajzel, P. Ruchat, C. Lucchesi ang G. Dietler, and E.B. Ringelstein, "Automatic classification of hits into artifacts or solid or gaseous emboli by a wavelet representation combined with dual gated tcd," Ultrasound in medecine and biology, vol. 32, pp. 2803-2809, 2001.

[5] K. Yosioka and Y. Kawasiwa, "Acoustic radiation pressure on a compressive sphere," Acoustica, vol. 5, pp. 167-173, 1955.

[6] T. Hasegawa and K. Yosioka, "Acoustic radiation force on a solid elastic sphere," JASA, vol. 46, pp. 1139-1143, 1969.

[7] P. Tortoli, M. Pratesi, and V. Michelasi, "Doppler spectra from contrast agents crossing an ultrasound field," IEEE trans. UFFC, vol. 47, pp. 716-725, 2000.

[8] J.M. Girault, D. Kouamé, G. Souchon, M. Biard, F. Vandermeulen, A. Bleuzen, and F. Tranquart, "Report on ability to differentiate gaseous and particulate matter using targeted microbubbles to microemboli.," Report of the UMEDS european project, 2005.

[9] M. Biard, Analyse du signal Doppler et instrumentation : Etude et caractérisation d'embole, $\mathrm{Ph}$. d thesis, Tours University, France, 2005. 